\title{
Influence of Gelatin Hydrogel Porosity on the Crystallization of $\mathrm{CaCO}_{3}$
}

\author{
Fitriana Nindiyasari, ${ }^{* \dagger}$ Lurdes Fernández-Díaz, ${ }^{*}$, , ${ }^{\dagger}$ Erika Griesshaber, ${ }^{\dagger}$ José Manuel Astilleros, $^{\S, \ddagger}$ \\ Nuria Sánchez-Pastor, ${ }^{\S}$ and Wolfgang W. Schmahl ${ }^{\dagger}$ \\ ${ }^{\dagger}$ Department of Earth and Environmental Sciences, Ludwig-Maximilians-Universität, 80333 Munich, Germany \\ ${ }^{\S}$ Departamento de Cristalografía y Mineralogía, Universidad Complutense de Madrid, 28040 Madrid, Spain \\ ${ }^{\ddagger}$ Instituto de Geociencias (UCM, CSIC), C/José Antonio Novais 2, 28040 Madrid, Spain
}

\begin{abstract}
We investigated the influence of the porosity of the growth medium on the crystallization of calcium carbonate in hydrogels with different gelatin solid contents $(2.5,5$, and $10 \mathrm{wt} \%)$. In all experiments, the precipitate consisted of calcite with occasional occurrences of some vaterite and aragonite. The calcite grew as compact radial intergrowths of crystals that show rhombohedral external faces. The crystal surfaces consist of identical 1-10 $\mu \mathrm{m}$ sized rhombohedral sub-blocks. Electron backscatter diffraction (EBSD) uncovered the radial inter-

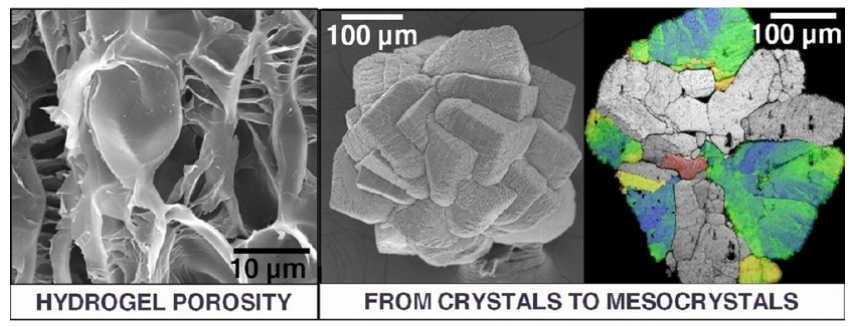
growth structure of the aggregates. EBSD also documented the internal microscale mosaicity and mesocrystal-like constitution of the gel-grown calcite. Raman spectroscopy and thermogravimetric analysis confirmed the presence of gelatin within the crystals. It reached up to $\sim 2$ mass $\%$ in the calcite-gelatin composites that formed in hydrogels with 10 wt \% gelatin content. Calcite morphology and mosaicity varied with the gelatin content of the hydrogel, such that an increase in gelatin content initiated the growth of smaller crystal aggregates having progressively rougher surfaces, increasing amounts of incorporated gel, and increasing degrees of misorientation in the internal mosaic structure. Apart from biospecific morphology, the gel growth experiment successfully mimics many characteristics of calcite biomineralization such as formation of a hierarchical hybrid composite, crystal mosaicity, and mesocrystal-like constitution.
\end{abstract}

\section{INTRODUCTION}

Crystal growth in hydrogel matrices is a long-stated method, and its advantages to model biomineralization become increasingly evident. ${ }^{1,2}$ Numerous authors have emphasized the role of organic matrices in the biomineralization processes in a variety of organisms (molluscs, corals, coccolithophorids, etc). ${ }^{3-5}$ Such matrices consist of assemblies of polysaccharides, proteins, and glycoproteins and show different active groups as well as different porosities. Moreover, it is well-established that most biomineralization processes occur in confined environments within organic matrices. ${ }^{6,7}$ These natural organic matrices show features strikingly similar to those of hydrogel-systems. The possibility of fine-tuning the porosity and the chemical characteristics of hydrogels by using different solid gel contents and additives in their preparation constitutes a further advantage of hydrogels for modeling biomineralization processes. ${ }^{8-11}$

Numerous authors have studied the crystallization of $\mathrm{CaCO}_{3}$ in a variety of hydrogels (silica, agar, gelatin, polyacrylamide, etc) ${ }^{12-17}$ In many cases, experiments yielded calcite crystals that exhibited a wide range of unexpected shapes (octahedron, cuboctahedron, dodecahedron, etc.). Such crystals were built up by nanometric to micrometric rhombohedral sub-blocks that are slightly tilted relative to each other. These crystal entities often incorporate small amounts (1-3 wt \%) of the hydrogel matrix that is present at the contact between the building subblocks. ${ }^{15-19}$ For these objects, descriptive terms such as "hybrid structure of calcite crystalline assemblies" 15 or "single-crystalline nanoclustered calcite of semicoherent domains"18 have been coined. More recently, these objects are described as "mesocrystals"., ${ }^{1,20-23}$ The definition of a mesocrystal evolved to the statement of Seto et al.: 22 "a mesocrystal ideally comprises a $3 \mathrm{D}$ array of isooriented single crystal particles of size 1-1000 $\mathrm{nm}$ " (with no reference to hybrid composite structure). Song and Cölfen ${ }^{24}$ assign mesocrystal formation in gels to nanoparticle alignment along an organic matrix, based on work by Grassmann and co-workers ${ }^{15-18}$ and Huang et al. ${ }^{19}$ Nanoparticle assembly processes or so-called nonclassical crystallization becomes an increasingly important concept in the crystallization of mesocrystals and in nucleation and growth of calcium carbonate. ${ }^{1,25-30}$ Carbonate biominerals in skeletons and teeth typically have characteristics of a hybrid (organic-inorganic) mesocrystalline assembly and a hierarchical architecture over several length scales. ${ }^{31-36}$ Grassmann et al. ${ }^{16}$ already described nanoparticle assembly as a nonclassical growth mode of calcite 
forming at high supersaturation in gels, a notion which has been suggested for general systems with very low molecular solubility or high supersaturation by Cölfen and Antonietti. ${ }^{23}$

Over the past decade, the influence on carbonate crystallization of both, the hydrogel types as well as the organic and inorganic additives, to the growth medium have been extensively explored. ${ }^{2,37-41}$ To the knowledge of the authors, only Helbig ${ }^{42}$ has studied the effect of the solid gel concentration of polyacrylamide hydrogels on the polymorph selectivity and morphology of $\mathrm{CaCO}_{3}$ crystals. It has been concluded that the solid content of the hydrogel has little to no effect on polymorph selectivity. ${ }^{42}$ However, it strongly affects the morphology of calcite crystals, which upon growth, evolves from rhombohedron to octahedron-shaped single crystals and crystal aggregates composed of oriented sub-blocks.

Gelatin is an interesting hydrogel, since its porosity can easily be modified by changing the solid content of the gel. In this study, gelatin hydrogels were used to conduct $\mathrm{CaCO}_{3}$ crystal growth experiments by using a double diffusion setup. The gels were prepared with three different gelatin contents: $2.5,5$, and 10 wt $\%$. One major aim of this work was to explore the influence of gelatin content on the nucleation of $\mathrm{CaCO}_{3}$ and to investigate the variation in experimental waiting time for the first optically visible crystal, the position of the first precipitate, the number of nuclei, and the type of polymorph formed. A further aim was to study the effect of gelatin content on the growth morphology of calcite crystals, focusing on possible differences in their mosaicity. The final goal of this work was to relate changes of $\mathrm{CaCO}_{3}$ precipitates to changes in porosity and diffusivity of the crystallization medium relative to the gelatin content of the hydrogel.

\section{EXPERIMENTAL PROCEDURES}

Crystal Growth Experiments. $\mathrm{CaCO}_{3}$ crystal growth experiments were conducted at $15{ }^{\circ} \mathrm{C}$ in a double-diffusion system consisting of two vertical branches separated by a horizontal column of hydrogel. At this temperature, the hydrogels remain rigid. Also, this temperature is within the range of common temperatures in shallow sea waters, ${ }^{43}$ the living environment for a variety of organisms. ${ }^{44-46}$

The reagents, aqueous solutions of calcium chloride $\left(0.5 \mathrm{M} \mathrm{CaCl}_{2}\right.$; Sigma Aldrich) and sodium carbonate $\left(0.5 \mathrm{M} \mathrm{Na}_{2} \mathrm{CO}_{3}\right.$; Sigma Aldrich), filled the vertical branches from where they counter-diffused through the porous structure of the hydrogel. The hydrogel was prepared by dissolving porcine gelatin (Sigma Aldrich; Type A, Bioreagent) in highpurity, deionized (Milli- $\mathrm{Q}$ ) water $\left(18.2 \mathrm{M} \Omega\right.$ ) heated at $60{ }^{\circ} \mathrm{C}$. The gelification was carried out at $4{ }^{\circ} \mathrm{C}$ for an hour, and the hydrogels were subsequently set at $15^{\circ} \mathrm{C}$ for $24 \mathrm{~h}$ before pouring the reagent solutions in the vertical branches. The hydrogels were prepared using three different contents of gelatin: $2.5,5$, and $10 \mathrm{wt} \%$. The hydrogel column was $70 \mathrm{~mm}$ long and had a diameter of $9 \mathrm{~mm}$ in all the cases. At the start of the experiments, the counter-diffusion of the reagents determined that eventually $\mathrm{CaCO}_{3}$ nucleation and growth occurred in a narrow region of the hydrogel column. The time $\left(t_{\mathrm{w}}\right)$ that elapsed between the start of the experiments and the occurrence of the first crystals that were detected with $400 \times$ magnification was measured as a means to determine differences in physicochemical conditions in the experiments during early crystallization stages. It is important to note that, although the experimental waiting time for the first optically visible crystal is always longer than the actual nucleation time and is influenced by the method used to detect the first nuclei, the error involved in its measurement is identical in all experiments, which makes this parameter $\left(t_{\mathrm{w}}\right)$ suitable for comparison between the different experiments. Crystal growth was monitored by optical microscopy. One week after the observation of the first optically visible crystals, the experiments were stopped and the crystals were extracted by dissolving the gel in hot water and filtering the precipitate through a $1 \mu \mathrm{m}$ pore-sized membrane. The crystals were subsequently washed 3 times with Milli-Q water heated at $60^{\circ} \mathrm{C}$ to remove any impurity and dried in air at room temperature. The entire extraction/washing procedure lasted $\sim 15 \mathrm{~min}$. In order to ensure that this procedure does not influence the morphological characteristics nor the surface features of crystals, we have conducted the following experiments:

Reference crystals with rhombohedral faces were prepared (i) by crushing a Spar quality calcite single crystal and sieving to select small fragments of the desired size, (ii) by growing calcite rhombohedra in water by the gas-diffusion method. SEM images of the crushed crystals without further treatment and water-grown crystals, which were washed in alcohol and rapidly dried, were obtained. The gel extraction procedure (washing at $60{ }^{\circ} \mathrm{C}$ for $15 \mathrm{~min}$ ) was then applied to the reference crystals, and those sample were SEM-imaged similarly. The crushed calcite crystals were bounded by flat (104) faces, the calcite cleavage plane, as expected. The only feature that could be distinguished by SEM on their surface, irrespective of their having been in contact with the hot water or not, was the presence of cleavage steps that formed during the crushing. Calcite crystals obtained by gas diffusion in (ii) showed the typical rhombohedral habit, with flat (104) faces and straight edges. No differences were detected by SEM between crystals that had been in contact with hot water and those that had not. In summary, no SEM-visible etch pits formed on the crystal surface of calcite as a result of their being in contact with $60{ }^{\circ} \mathrm{C}$ water for $15 \mathrm{~min}$ (Figure $\mathrm{S} 1$ of the Supporting Information).

Qualitative Analysis of the Gel Porosity. In order to obtain qualitative information on the porosity of the hydrogel as a function of its gelatin content, small volumes $(30 \mathrm{~mL})$ of hydrogel with different gelatin contents were prepared following the procedure described for the growth experiments. Hydrogel samples were frozen with liquid nitrogen and subsequently lyophilized during $24 \mathrm{~h}$ in a lyophilizer (Lyoquest-55 Eco, Telstar) to eliminate all the water trapped in the gel pores. Fragments of the dried samples were double coated first with carbon and subsequently with gold. Scanning electron microscope images were obtained with a JEOL JSM 6400 operated at $20 \mathrm{kV}$. Since it is expected that the lyophillization process alters the porosity of the samples, these observations are exclusively used for comparison purposes.

Reagent Concentrations at Nucleation Time and Location. In order to obtain information on the concentration of the reagents at the experimental waiting time for the first optically visible crystal in the region of the hydrogel column where the first crystals formed, two sets of independent experiments were conducted. In both cases, one of the vertical branches of the experimental setup was filled with water, while the other was filled with one of the reagent solutions. Independent runs were conducted for each content of solid gelatin in the hydrogel. The experiments were stopped after times equal to the time delayed between the beginning of the corresponding growth experiment and the observation of the first crystals: $\sim 24 \mathrm{~h}$ for the hydrogel with a $2.5 \mathrm{wt}$ $\%$ solid content, $\sim 84 \mathrm{~h}$ for the hydrogel with a $5 \mathrm{wt} \%$, and $\sim 120 \mathrm{~h}$ for the hydrogel with 10 wt $\%$ gelatin content. The gelatin column was then recovered and the region of this column corresponding to the location of the first nuclei was cut, dissolved in water, and analyzed. Ca analyses were conducted by inductively coupled plasma-optic emission spectrometry (ICP-OES) using an ICP-Arcos (spectrolab). The concentration of carbonate ions was determined by potentiometry, using a $\mathrm{pH}$-meter Orion 710A. The information obtained in this way allowed us to estimate the relative supersaturation at an experimental waiting time of the first optically visible crystal in the region of the hydrogel column where the first nuclei were detected and correlate it to the gelatin solid content of each hydrogel. This calculation was conducted using the geochemical speciation code PHREEQC ${ }^{47}$ and the PHREEQC.DAT database.

$\mathrm{CaCO}_{3}$ Crystals Characterization. The crystals obtained were coated with carbon and imaged using a FEG-SEM (JEOL JSM 6400) operated at $20 \mathrm{kV}$. Selected crystals were characterized using X-ray powder diffraction (XRD). The XRD analyses were carried out on a Philips X'Pert Pro X-ray diffractometer in the $2 \theta$ angle range between $5^{\circ}$ and $80^{\circ}$ in steps of $0.02^{\circ}$ using $\mathrm{Cu} \mathrm{K} \alpha$ radiation. Raman spectra of the samples were obtained using a confocal Thermo Fischer DXR Raman 
Microscope. It allowed us to establish the correlation between a given crystal morphology and a specific $\mathrm{CaCO}_{3}$ polymorph. The objective selected was of $10 \times$ together with a laser source $780 \mathrm{~nm}$ at $10 \mathrm{mV}$ in a laser mode power at $100 \%$. The average spectral resolution of the Raman shift ranging from 70 to $3000 \mathrm{~cm}^{-1}$ was $2-4 \mathrm{~cm}^{-1}$ (i.e., grating 400 lines/ $\mathrm{mm})$. Operated under OMNIC 1.0 with a pinhole aperture of $50 \mu \mathrm{m}$ and a bleaching time of $1-2 \mathrm{~s}, 4$ to 8 exposures of 10 to $15 \mathrm{~s}$ were averaged, depending on the sample. Thermogravimetric and differential thermal analyses (TGA-DTA) were performed using a SDT-Q600 apparatus on calcite samples to detect the possible incorporation of the different amounts of gelatin as a function of the gelatin solid content in the hydrogel. The weight loss between room temperature and $150{ }^{\circ} \mathrm{C}$ was considered the consequence of drying. The weight loss corresponding to the loss of gelatin was considered to start at $200{ }^{\circ} \mathrm{C}$.

EBSD measurements were performed on embedded and highly polished crystal surfaces. The required highly even surface of the samples was achieved by a final etch-polishing with colloidal silica in a vibratory polisher. The samples were coated with $4 \mathrm{~nm}$ of carbon. EBSD maps were obtained at $20 \mathrm{kV}$ on an FEG-SEM (JEOL JSM 6400) equipped with an Oxford Instruments NordlysNano EBSD detector.

\section{RESULTS}

Hydrogel Porosity. During their preparation for SEM imaging, the hydrogels undergo a certain shrinkage associated with the elimination of the water trapped in their porous structure. This shrinkage was similar in all samples $(\sim 17$ wt $\%$ volume reduction) and affected mainly their outer region. In order to guarantee a minimum effect of this shrinkage with the porous structure of the hydrogels, all the SEM images were collected on fresh fracture surfaces on the core of the samples. The comparison between SEM images obtained on samples with different gelatin content clearly indicates that the concentration of gelatin strongly influences the porosity structure of the hydrogels. This influence is reflected by a progressively more complex porosity as the gelatin content increases and is also evidenced by changes in both the pore sizes and pore wall thickness (Figure 1). Thus, hydrogels with $2.5 \mathrm{wt} \%$ gelatin show pore sizes $>10 \mu \mathrm{m}$ (Figure 1, panels a and $\mathrm{d}$ ), whereas in hydrogels with solid-gel contents of 5 and $10 \mathrm{wt} \%$ pore sizes are $\leq 5 \mu \mathrm{m}$ (Figure 1, panels b, c, e, and f). Moreover, the thickness of

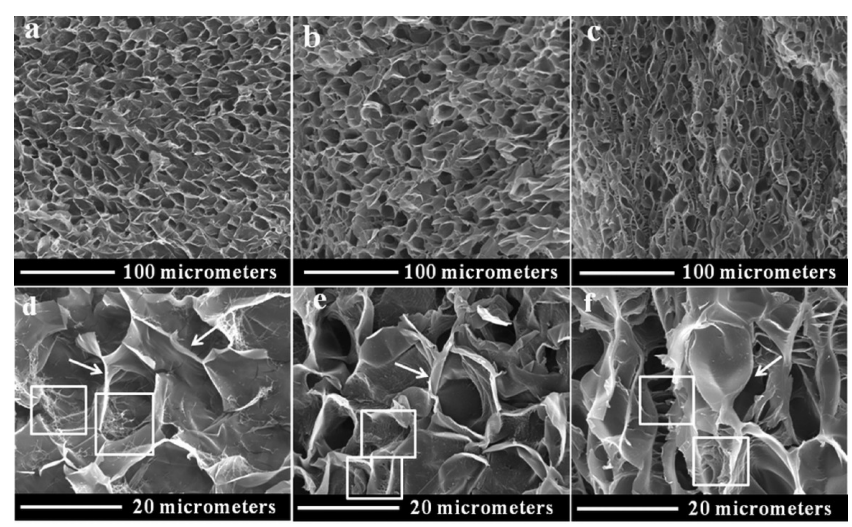

Figure 1. SEM images illustrating the variation of the porosity of gelatin hydrogels as a function of their solid content. Images $(a-c)$ show a general overview of this porosity when the gelatin content was $2.5,5$, and $10 \mathrm{wt} \%$, respectively. Images $(\mathrm{d}-\mathrm{f})$ show closeups of the pores in $(\mathrm{a}-\mathrm{c})$, respectively. A progressive reduction of the pores size with increasing gelatin content in the hydrogel is observed. This reduction correlates with a thickening of the walls, separating the pores and an increase of the complexity of the porosity structure. White squares in images $(d-f)$ highlight the existence of small pores on the walls interconnecting larger pores. White arrows highlight the thickness of the interpore walls. the pore walls clearly increases with higher gelatin content in the hydrogel. These walls are very thin and show a spiderweb-like structure (see areas within the square frames in Figure 1d) in hydrogels with 2.5 wt \% gelatin content. Pore walls in hydrogels with 5 wt \% gelatin content are thicker. Although in this case the spiderweb-like structure shows a poorer development, each pore seems to contain several subpores of smaller sizes (see areas within the square frames in Figure 1e), which appear located on the walls separating bigger pores. Finally, pore walls in hydrogels with 10 wt \% solid gel content are significantly thicker and show no development of a spiderweb-like structure. It is important to note that in this case, a complicated arrangement of thinner walls develops within the pores. These thinner walls connect opposite sides of bounding-pore walls and subdivide the bigger pores in cages with sizes in the range between 1 and $2 \mu \mathrm{m}$ (see areas within the square frames in Figure 1f).

Influence of the Gelatin Content on the Early Stages of Crystallization and Polymorph Selectivity. Table 1 summarizes the experimental waiting time for the first optically visible crystals $\left(t_{\mathrm{w}}\right)$, the location of these crystals in the hydrogel column (distance measured from $\mathrm{CaCl}_{2}$ reservoir), the number of crystals (NC), and the type of polymorph observed. The

Table 1. Experimental Waiting Time for First Optically Visible Crystal $\left(t_{\mathrm{w}}\right)$, Location of the First Crystals in the Gel Column (Distance Measured from $\mathrm{CaCl}_{2}$ Reservoir) and Total Number of Crystals (NC) of Calcite (Cal), Vaterite (Vtr) and Aragonite (Arg) Individuals Measured $24 \mathrm{~h}$ after the Observation of the First Crystal

\begin{tabular}{cccccc} 
& & & \multicolumn{3}{c}{$\mathrm{NC}$} \\
\cline { 3 - 6 } wt \% gelatin & $t_{\mathrm{w}}$ (hours) & position $(\mathrm{mm})$ & $\mathrm{Cal}$ & $\mathrm{Vtr}$ & Arg \\
2.5 & $\sim 24$ & $32-35$ & 70 & 28 & 0 \\
5 & $\sim 84$ & $37-39$ & 32 & 6 & 1 \\
10 & $\sim 120$ & $38-41$ & 17 & 3 & 1 \\
\hline
\end{tabular}

experimental waiting time for the first optically visible crystal progressively increased with the gelatin content in the hydrogel, being $\sim 24 \mathrm{~h}$ for $2.5 \mathrm{wt} \%$ gelatin content, $\sim 84 \mathrm{~h}$ for $5 \mathrm{wt} \%$ gelatin content, and $\sim 120 \mathrm{~h}$ for $10 \mathrm{wt} \%$ gelatin content, respectively. In all experiments, the crystals first appeared in a narrow, central region $(\sim 5 \mathrm{~mm})$ of the hydrogel column. An increase in gelatin content caused the displacement of the location of the first optically visible crystals toward the calcium reservoir. With increased gelatin concentration, the number of crystals in the first visible precipitate decreased. In our experiments, the hydrogel column remained transparent and did not show any shade change before the observation of the first crystals. The first optically visible crystals always were calcite irrespective of the gelatin content of the hydrogel. Moreover, in all experiments calcite remained the main component of the precipitate. In the final stages of crystallization some vaterite was also present. We could observe a distinct negative correlation between the amount of vaterite and the gelatin content of the hydrogel. This strongly implies that gelatin inhibits the crystallization of vaterite. While vaterite represented $\sim 10 \%$ of the precipitate in the experiments conducted using hydrogels with a 10 wt \% gelatin content, its amount increased up to $\sim 40 \%$ when the hydrogel contained 2.5 wt $\%$ gelatin (see Table 1 ). Aragonite was not observed in the experiments conducted with $2.5 \mathrm{wt} \%$ gelatin hydrogel. It was detected as a minor phase when the gelatin content in the hydrogel was 5 and $10 \mathrm{wt} \%$. 
Influence of the Gelatin Content on Crystal Size and Morphology. The size of the crystals was measured 7 days after their first observation. Vaterite grew as spherulitic aggregates with a mean size of $\sim 100 \pm 20 \mu \mathrm{m}$. These aggregates consisted of small lens-shaped individuals $(\sim 10 \times 1 \mu \mathrm{m})$ in a rosettelike arrangement (Figure 2a). The gelatin content of the hydrogel did
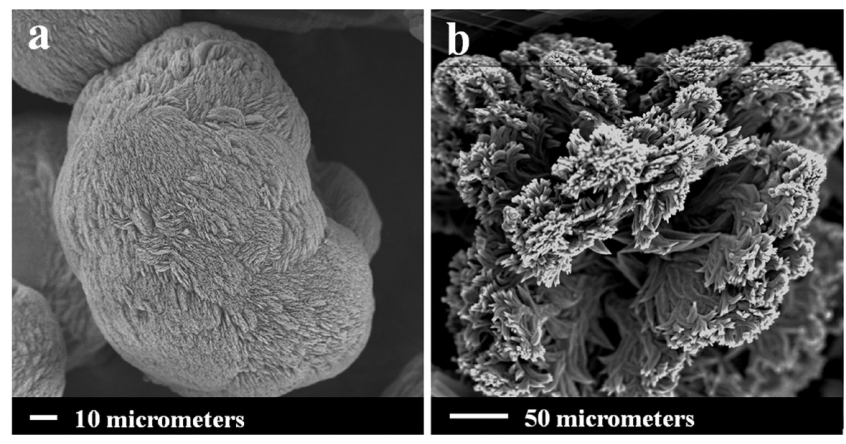

Figure 2. (a) Spherulitic aggregates of vaterite and (b) radially growing fibrous aragonite.

not affect the size of the vaterite crystals. Aragonite grew as aggregates consisting of radiating, fibrous crystals (Figure $2 b$ ). In the case of calcite, both the morphology and the size of the crystal changed significantly with the gelatin content of the hydrogel. Calcite grew in hydrogels with 2.5 wt \% gelatin content as blocks formed by several subparallel crystals bounded by flat, terraced (104) surfaces (Figures 3, panels a and b) and had a mean size of $220 \pm 70 \mu \mathrm{m}$. Calcite grown in hydrogels with 5 wt $\%$ gelatin content appeared as both blocks, as above, and radial aggregates composed of small and blocky rhombohedra. These crystals were bounded by strongly terraced (104) as well as rough, rounded vicinal surfaces (Figures 3, panels $\mathrm{c}$ and $\mathrm{d}$ ). The mean size of the calcite aggregates was $400 \pm 50 \mu \mathrm{m}$. Single crystals that were elongated parallel to their $c$ axis were also observed (Figure $3 \mathrm{e}$ ). These crystals also showed flat (104) but also rough, curved surfaces that seem to belong to the prism region. In these rough surfaces, crystalline sub-blocks were present (Figure $3 \mathrm{f}$ ) that were slightly tilted relative to each other. Calcite grown in hydrogels with $10 \mathrm{wt} \%$ gelatin content formed crystal aggregates of numerous individuals. The mean size of these aggregates was $\sim 500 \mu \mathrm{m}$. Their constituting individuals had sizes around 100 $\mu \mathrm{m}$ and were bounded by rough (104) faces with curved, rough edges and corners (Figure $3 \mathrm{~g}$ ). The increased roughness in regions near the crystal edges is depicted in Figure 3h, where the presence of the tilted crystalline sub-blocks can be observed. Each sub-block, ranging from less than $1 \mu \mathrm{m}$ to over $5 \mu \mathrm{m}$ in size, shows the typical morphology of the calcite rhombohedron (Figure 4a). An interesting example of the rhombohedral subblocks forming the calcite crystal aggregates grown in a hydrogel with 10 wt \% gelatin is shown in Figure $4 \mathrm{~b}$. The rhombohedronshaped sub-blocks seem to be hierarchically arranged, with their dimensions covering several size ranges. A comparison of crystallite size determined from XRD and the Scherrer equation (without corrections for stress) to selected hkl reflections with the pixel-step of the EBSD measurements is shown in Table $S 1$ of the Supporting Information.

Influence of Hydrogel Gelatin Content on Crystal Morphology and Mosaicity. Figures 5 and 6 show EBSD (electron backscatter diffraction) maps and corresponding pole figures of crystal aggregates that grew in hydrogels with different gelatin content. Figures 5a and 6a show crystals obtained from

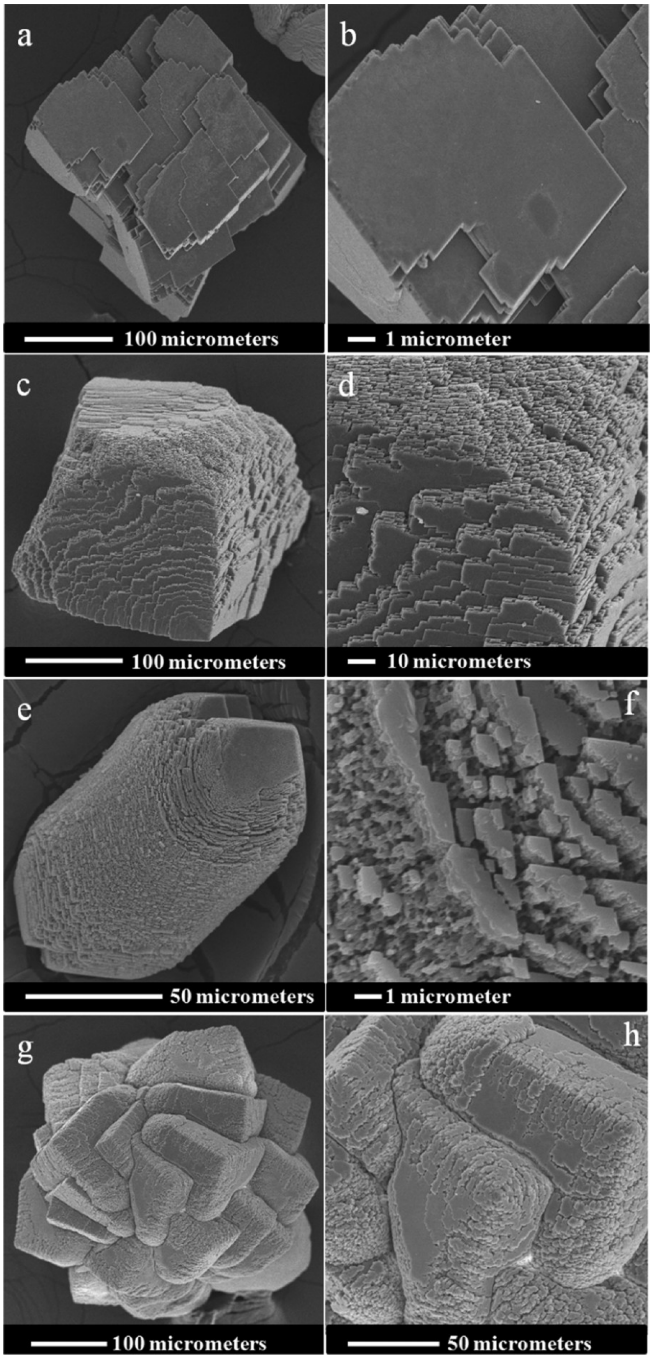

Figure 3. (a) Blocky calcite bounded by (104) surfaces grown in a hydrogel with $2.5 \mathrm{wt} \%$ gelatin content. (b) Detail of the surface, with flat appearance. (c) Calcite crystal grown in a hydrogel with 5 wt \% gelatin content, bounded by (104) surfaces and nonsingular surfaces. (d) Detail showing the formation of curved surfaces due to the accumulation of rough steps. Note that, while (104) surfaces are relatively flat, other surfaces are very rough. (e) Calcite crystal grown in a hydrogel with $5 \mathrm{wt}$ $\%$ gelatin content. The crystal is elongated along the $c$ axis. (f) Detail showing the high roughness of the curved surfaces. $(\mathrm{g})$ Calcite crystal aggregate grown in a hydrogel with $10 \mathrm{wt} \%$ gelatin content. (h) Close up of $(\mathrm{g})$, showing the higher roughness of the surfaces near the edges.

hydrogels containing $2.5 \mathrm{wt} \%$ gelatin, and Figures $5 \mathrm{~b}$ and $6 \mathrm{~b}$ display crystals that were obtained from hydrogels with $10 \mathrm{wt} \%$ gelatin content. Crystallographic calcite orientation patterns are given color-coded in both the EBSD maps and the corresponding pole figures. Superimposed on the color in the map is the EBSD band contrast (Figure 5, panels a and b). This is a gray scale component that gives the signal strength in each individual EBSD-Kikuchi diffraction pattern. Where carbonate mineral is present, the strength of the EBSD pattern is high, while in pores and gel inclusions, the strength of the EBSD pattern is low. The band contrast signal thus indicates the distribution of incorporated gelatin within the crystal. Also shown in Figure 5 is the mean calcite orientation for all crystals composing the investigated aggregates. For crystal aggregates grown in hydrogels with $10 \mathrm{wt} \%$ gelatin, the orientation of the constituting crystals shows random scatter (Figure 5b), while for the crystal 


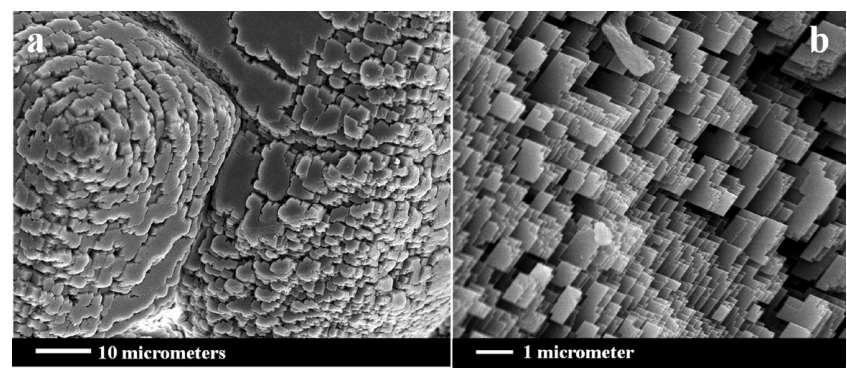

Figure 4. Images of the surface of calcite crystals grown in a hydrogel with $10 \mathrm{wt} \%$ gelatin content. An increased surface roughness is present and indicates that the crystal aggregates consist of rhombohedral subblocks arranged in an approximately equal orientation. (a) Near the edges of the crystal surface, roughness is increased. (b) The size of the rhombohedral sub-blocks varies between 1 to $5 \mu \mathrm{m}$.

aggregates grown in hydrogels with 2.5 wt \% gelatin (Figure 5a) some degree of mutual co-orientation of the constituting crystals is present. This correlates with the vaguely rhombohedral overall shape of the aggregates grown in hydrogels with $2.5 \mathrm{wt} \%$ gelatin.

We observe significant differences in the morphology of the crystal aggregates grown in the two different media. An increase in hydrogel gelatin content causes rounded crystal surfaces (Figure 6b) and a pronounced internal mosaic structure of each crystal in the aggregate (i.e., each crystal is composed of several microscale subcrystals).

Incorporation of Gelatin into Calcite Crystals during Growth and Mesocrystallinity. In order to study the incorporation of gelatin into the calcite crystals during their growth, Raman spectra were collected on three selected calcite samples. The spectra are shown in Figure 7. The Raman spectrum of calcite is characterized by two low frequency bands at 155 and $281 \mathrm{~cm}^{-1}$, which arise from external vibrations and three higher frequency bands at 711,1085 , and 1435 , resulting from the $\mathrm{CO}_{3}{ }^{2-}$ group's internal vibrations. ${ }^{48-51}$ All these bands are clearly identifiable and show identical characteristics in the spectra of the three studied calcite samples. Most interesting is the presence of weak bands around 488,1016, 1341, 1748, and $2952 \mathrm{~cm}^{-1}$ in all three spectra. Although the band at $1748 \mathrm{~cm}^{-1}$ may be interpreted as resulting from the combination of calcite bands $\nu_{1}$ and $\nu_{4},{ }^{52}$ the other bands cannot be assigned to any vibration in the calcite structure. However, some of these bands occur at wavelengths that indicate gelatin in the calcite crystals. The Raman spectrum that was collected on the powdered porcine gelatin that was used to prepare the hydrogels is shown in Figure S2 of the Supporting Information. For example, the sharper band around $2900 \mathrm{~cm}^{-1}$, present in the spectrum of the powdered gelatin, is commonly assigned to the $\mathrm{CH}$ stretching mode and is considered indicative of the presence of organic material. ${ }^{53}$ Most significant is the band located around 1016 $\mathrm{cm}^{-1}$, that is present in both the calcite crystals and the powdered porcine gelatin spectra. Bands in the region between 1005-1020 $\mathrm{cm}^{-1}$ occur in the spectra of different gelatins. ${ }^{54}$ They have also been found in the shells of some marine mollusks, ${ }^{55}$ lobster shell pigments, ${ }^{56}$ birds feathers, ${ }^{57}$ and are indicative of the presence of methyl groups, either as a $\mathrm{CH}$ out-of-plane ${ }^{58}$ or as the deformation-activated $\mathrm{CH}=\mathrm{CH}$ wagging modes. ${ }^{53}$ The increasingly stronger intensity of the $1016 \mathrm{~cm}^{-1}$ band in the calcite spectra (see inset in Figure 7) supports the interpretation that gelatin is incorporated into calcite crystals and that this incorporation is much more marked as the hydrogel gets heavier.

Thermogravimetric analyses (TGA) and differential analyses (DTA) of calcite samples grown in hydrogels with different gelatin solid content also supported the incorporation of small amounts of organic hydrogel into the calcite crystal aggregates (Figure S3 of the Supporting Information). This amount varied as a function of the gelatin concentration in the hydrogel, being extremely small, around 0.2 mass \%, for crystals grown in hydrogels with $2.5 \mathrm{wt} \%$ gelatin. It increased to 0.9 and 1.5 mass \% for crystals formed in hydrogels with 5 and 10 wt \% gelatin contents, respectively. These values are consistent with the 1.9 mass \% of gelatin content determined by TGA in calcite mesocrystals grown at $25{ }^{\circ} \mathrm{C}$ in a gelatin hydrogel with a $10 \mathrm{wt} \%$ gelatin solid content ${ }^{(19)}$ and are similar to the estimated amount of organic hydrogel network (0.7 mass \%) incorporated into calcite grown in a polyacrylamide hydrogel. ${ }^{16}$ Details on the characteristics of TGA/DTA analyses of the different calcite samples can be found in Figure S3 of the Supporting Information.

High-resolution EBSD allows the investigation of crystallite and particle arrangement with a spatial resolution in the submicrometer scale range. ${ }^{59,60}$ Figure 8 shows one crystal in an aggregate grown in a hydrogel with $10 \mathrm{wt} \%$ gelatin. Well visible in the band contrast map of Figure $8 \mathrm{c}$ are traces of incorporated gelatin. These appear as gray lines and green and

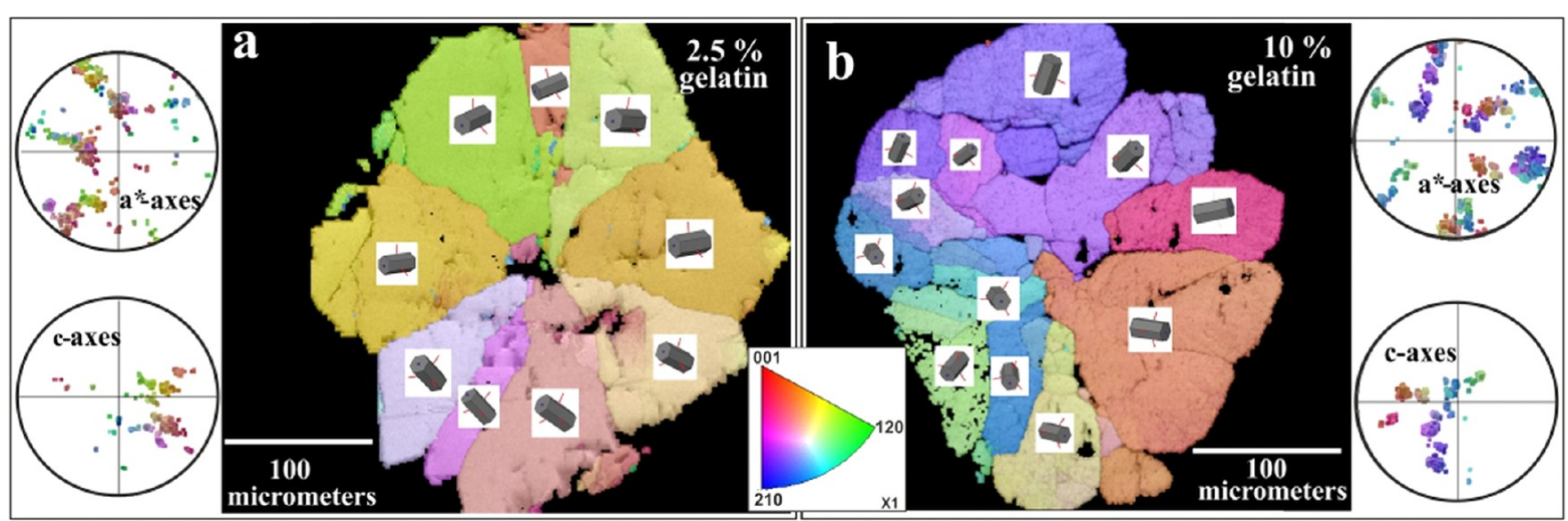

Figure 5. The influence of hydrogel gelatin content on the morphology of crystal aggregates. Figure 5a: crystal aggregate grown in a hydrogel with 2.5 wt $\%$ gelatin. Figure $5 \mathrm{~b}$ : crystal aggregate grown in a hydrogel with $10 \mathrm{wt} \%$ gelatin content. Crystal orientation patterns are given color-coded, in both, the EBSD maps as well as in the corresponding pole figures. 


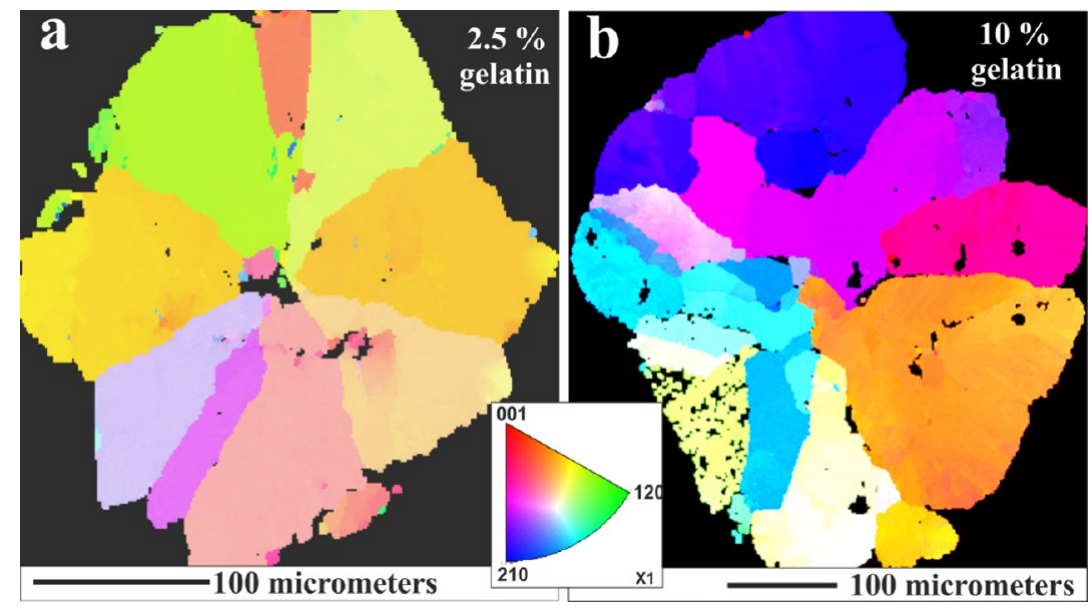

Figure 6. Difference in the degree of mosaicity of crystals, composing a crystal aggregate grown in hydrogels with 2.5 and 10 wt \% gelatin solid contents. (a) While the $\sim 100 \mu \mathrm{m}$ sized crystals composing the aggregate grown with $2.5 \mathrm{wt} \%$ gelatin content show very little internal structuring, (b) all crystals composing the aggregate grown with a gelatin content of $10 \mathrm{wt} \%$ display an increased internal substructuring (mosaic structure).

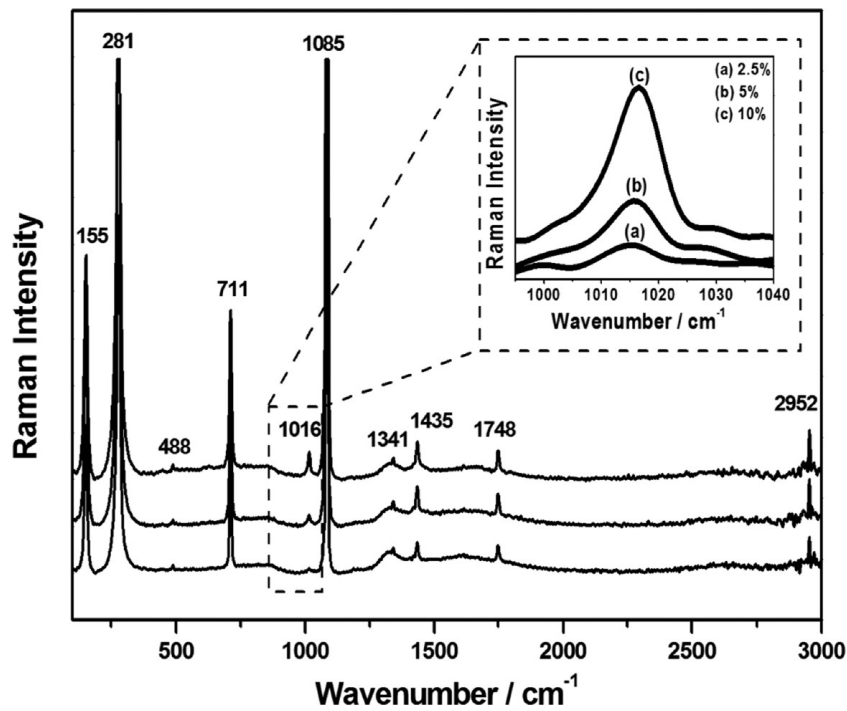

Figure 7. Raman spectra of three calcite samples grown in gelatin hydrogels with different gelatin solid content. The inset highlights the increasing intensity of the band at $1016 \mathrm{~cm}^{-1}$ as the gelatin solid content in the hydrogel increases. Raman spectrum of the powdered skin pork gelatin used to prepare the hydrogels is provided as Figure S2 of the Supporting Information.

black dots within the crystal. The variation of colors (which code for crystal orientation) in Figure 8 (panels a and d) clearly indicates that the crystal is composed of a multitude of mosaic blocks, each having a slightly different orientation (Figure 8 , panels a, e, and f). For the same crystal, Figure 9 highlights calcite misorientation variations along five trajectories within the crystal (Figure 9, panels a-e) and displays the overall statistics of internal misorientation of all measured EBSD pixels with respect to the average orientation of this particular crystal (Figure 9h). The misorientation in degrees also defines the color scale of the maps. Misorientation in the crystal ranges up to 6 degrees (Figure 9h). It is not evenly distributed. In the center of the crystal, the orientation happens to be identical to the average orientation (blue colors), while misorientation generally increases toward the rims. The misorientation profiles (Figure 9, panels a-e) indicate that misoriented mosaic blocks exist on the scale of tens of micrometers down to one micrometer or

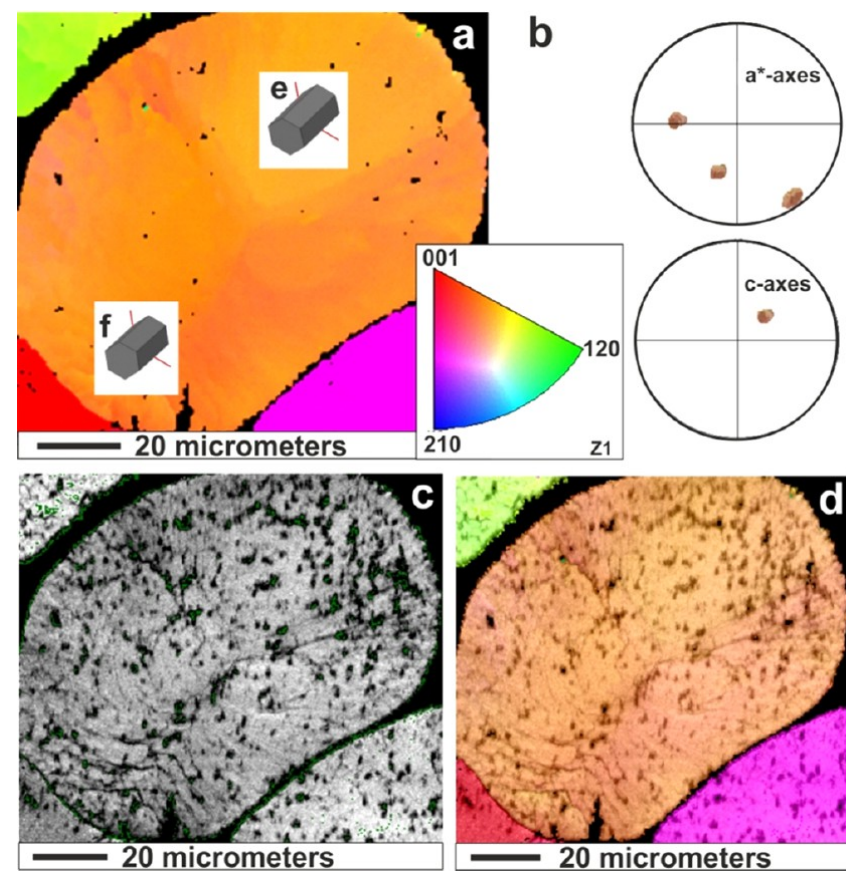

Figure 8. The mosaicity and hybrid composite constitution of a crystal in an aggregate grown from a hydrogel with $10 \mathrm{wt} \%$ gelatin. (a) A colorcoded EBSD map and (b) corresponding pole figure. (c) The band contrast image of the crystal highlights the incorporation of gelatin. (d) Map showing superimposed color-coded orientation and band contrast. (e and f) show the difference in the two major orientations that are encountered in this crystal.

below. The microscale mosaic blocks are usually separated by sharp small-angle boundaries associated with steps in the misorientation profiles. Moreover, a close inspection by zooming into local areas of the map (e.g., 9g, i) reveals a misorientation mosaic structure on the length scale of the $400 \mathrm{~nm}$ step size of the present EBSD measurement (i.e., the pixel size in the maps of Figure 9, panels f, g, and i). Accordingly, the crystal is constituted of an array of nanocrystallites which are imperfectly co-oriented. We may speculate that the imperfect co-orientation of the nanoscale building blocks is related to the presence of incorporated gel matrix not only on the microscale (as visible in Figure 8) but also on the nanoscale (Figure 9). Such a structure has been described 

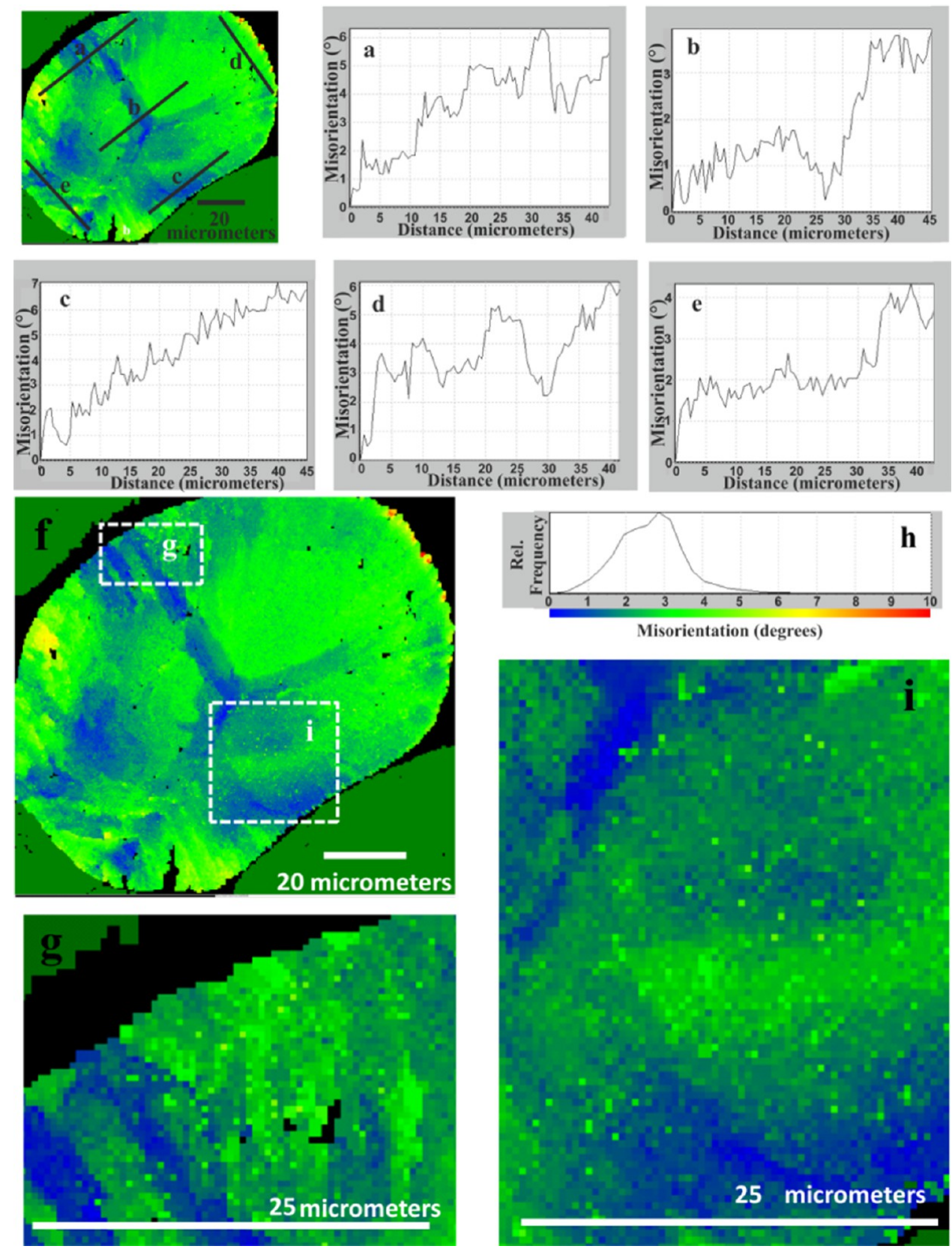

Figure 9. Profile lines showing local misorientation versus position within a crystal in a crystal aggregate grown in a hydrogel with $10 \mathrm{wt} \%$ gelatin content (Figure 9, panels a-e). ( $\mathrm{f}-\mathrm{i}$ ) Internal misorientation is displayed as color-coded maps and overall statistics (histogram $\mathrm{h}$ ) over the complete crystal shown in $\mathrm{f}$. The misorientation between microscale mosaic blocks composing the crystal reaches up to 6 degrees. (g and i) Note also the mesocrystal-like constitution indicated by the pixel-to-pixel misorientation shown in the enlarged regions; EBSD pixel (step) size is $400 \mathrm{~nm}$, and experimental orientation resolution is $\pm 0.3^{\circ}$.

as mesocrystalline (cf. Seto et $\mathrm{al}^{22}$ for the definition of a mesocrystal). The mesocrystal architecture allows continuous orientation gradients as can be seen in Figure 9c. Crystallite size estimation from XRD line broadening is given in Table $S 1$ and it supports the mesoscale structure on a length scale consistent with the EBSD observation.

Figure 10 compares the internal microscale mosaicity within the crystals composing a crystal aggregate grown in a hydrogel with 2.5 and 10 wt \% gelatin content, respectively. Well visible is the difference in internal structuring as well as internal misorientation. In the calcite grown in a hydrogel matrix with 10 wt \% gelatin, the overall mosaic spread is more than three times as high (up to 6 degrees) as the internal misorientation (about 1 degree) within the calcite of a crystal aggregate grown in a hydrogel with $2.5 \mathrm{wt} \%$ gelatin. These results clearly show the effect of gelatin content of the hydrogel matrix on $\mathrm{CaCO}_{3}$ crystallization and crystal aggregate formation. Note the almost dendritelike, branching mosaic structure in the sample from the gel with 10 wt $\%$ gelatin.

Physicochemical Conditions at Early Crystallization Stages. Table 2 summarizes the measured $\mathrm{pH}, \mathrm{Ca}^{2+}$ and $\mathrm{CO}_{3}{ }^{2-}$, concentrations for the diffusion experiments conducted with only one ion species for the estimation of the supersaturation at the experimental waiting time in the portion of the gel column where the first optically visible crystals were detected. Table 2 gives the calculated activities and nominal supersaturation levels 

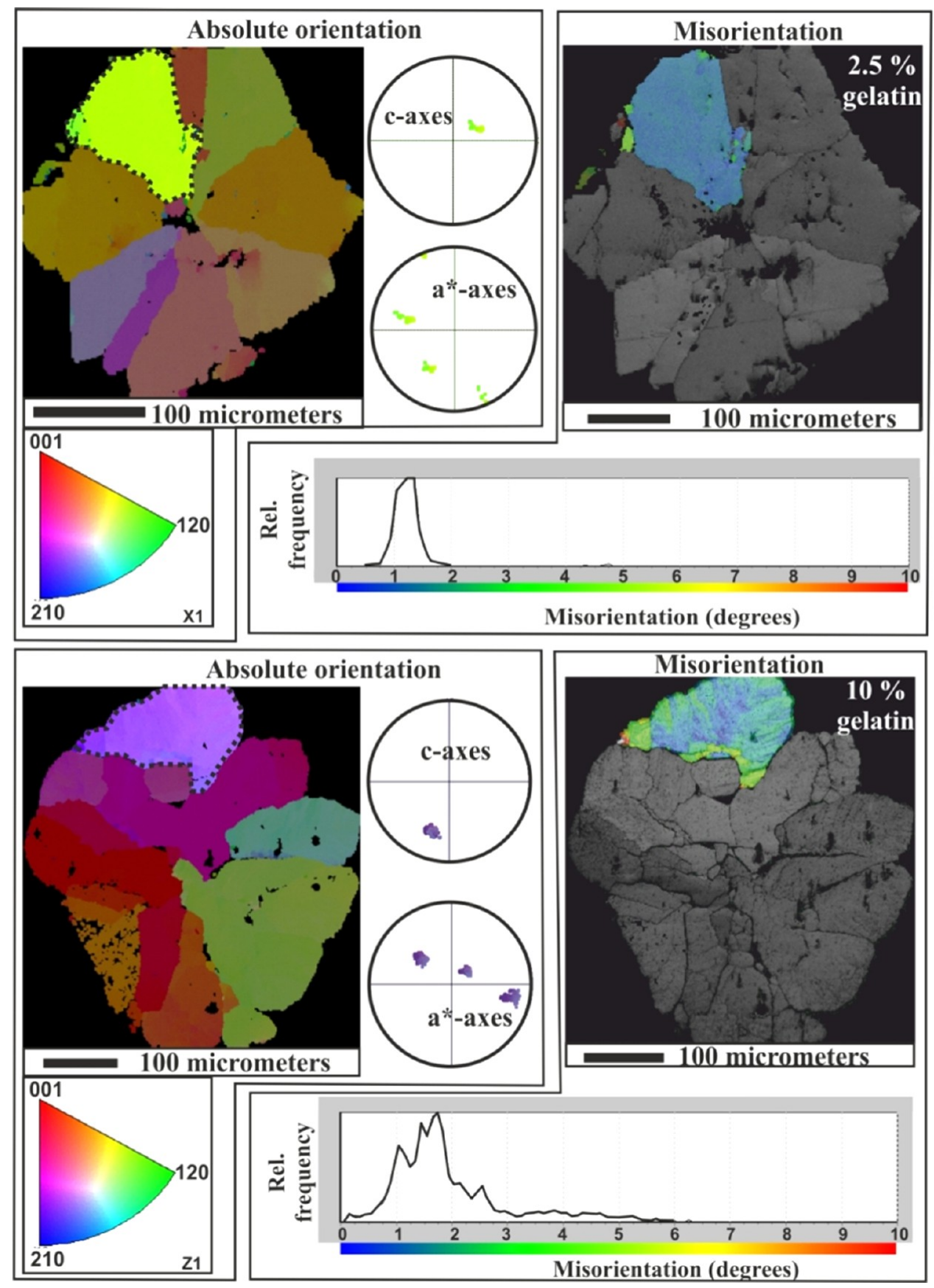

Figure 10. The effect of hydrogel gelatin content on the degree of calcite misorientation in the crystals composing a crystal aggregate grown in a hydrogel with 2.5 and 10 wt \% gelatin content, respectively. The mosaic spread (misorientation) in the crystals composing a crystal aggregate that grew in a hydrogel with $10 \mathrm{wt} \%$ gelatin is more than three times as high as that of crystals composing a crystal aggregate that grew in a hydrogel with $2.5 \mathrm{wt} \%$ gelatin. Note the branching, almost dendrite-like mosaic blocks which are separated by small-angle grain boundaries in the misorientation map.

Table 2. Summary of the Fluid Chemistry in the Hydrogel Pores at Experimental Waiting Time for the First Visible Crystal: pH, Total Concentration of Calcium and Carbonate $\left(\left[\mathrm{Ca}^{2+}\right],\left[\mathrm{CO}_{3}{ }^{2}\right]\right)$ in $\mathrm{mmol} / \mathrm{L}$, Activity of Calcium and Carbonate Ions $\left(a \mathrm{Ca}{ }^{2+}\right.$, $a \mathrm{CO}_{3}{ }^{2-}$ ), and Saturation Index and Saturation State with Respect to Calcite $\left(\mathrm{SI}_{\mathrm{Cal}}\right.$ and $\boldsymbol{\Omega}_{\mathrm{Cal}}$, respectively)

\begin{tabular}{cccccccc} 
gelatin solid content wt \% & $\mathrm{pH}$ & {$\left[\mathrm{Ca}^{2+}\right](\mathrm{mmol} / \mathrm{L})$} & {$\left[\mathrm{CO}_{3}{ }^{2}\right](\mathrm{mmol} / \mathrm{L})$} & $a \mathrm{Ca}^{2+}\left(\times 10^{-4}\right)$ & $a \mathrm{CO}_{3}{ }^{2-}\left(\times 10^{-2}\right)$ & $\mathrm{SI}_{\mathrm{Cal}}$ & $\Omega_{\mathrm{Cal}}$ \\
$2.5 \%$ & 9.64 & 5.10 & 42.47 & 2.66 & 1.137 & 2.9 & 794 \\
$5 \%$ & 9.84 & 48.02 & 97.24 & 17.03 & 2.022 & 3.9 & 7943 \\
$10 \%$ & 10.01 & 50.31 & 116.25 & 15.96 & 2.365 & 4.0 & 10000 \\
\hline
\end{tabular}

with respect to calcite. The nominal supersaturation is based on the assumption that no crystals or solid precursor phases are present. It is expressed in terms of the saturation index (SI) according to $\mathrm{SI}=\log (\Omega)=\log \left(\mathrm{IAP} / K_{\mathrm{sp}}\right)$, where ion activity product (IAP) is the product of the activities of the reacting ions (in this case, $\mathrm{Ca}^{2+}$ and $\mathrm{CO}_{3}{ }^{2-}$ ) powered to their stoichiometric 
number in the solid formula, $K_{\mathrm{sp}}$ (thermodynamic solubility product) is the value of IAP at thermodynamic equilibrium, and $\Omega=\mathrm{IAP} / K_{\mathrm{sp}}$ is the saturation state. The saturation indexes were calculated from $\mathrm{pH}$ and analytical chemical data of the aqueous solutions using PHREEQC3.0 and the PHREEQC.DAT database, in which the $K_{\mathrm{sp}}$ for calcite at $15^{\circ} \mathrm{C}$ is $10^{-8.3}$.

As it can be seen from Table 2, at the early stages of crystallization, increasing levels of supersaturation of the aqueous solution in the pores must be expected with increasing concentrations of gelatin in the hydrogel, unless an invisible precursor forms and buffers the supersaturation. Even though for all three cases the calculated nominal supersaturation is very high compared to situations where calcite forms in free solutions, it becomes extreme when the gelatin content in the hydrogel is 5 and $10 \mathrm{wt} \%$, respectively. At these stages, the nominal saturation state values are more than ten times higher than in hydrogels with 2.5 wt $\%$ gelatin at the time when the first, optically visible calcite crystals are detected.

\section{DISCUSSION}

Our results show that the solid content of the gelatin hydrogel has a slight influence on $\mathrm{CaCO}_{3}$ polymorph selection, with calcite being the main component of the precipitate in all the cases and vaterite representing about $40 \%$ of the precipitate in 2.5 wt $\%$ gelatin hydrogels and only $10 \%$ in $10 \mathrm{wt} \%$ gelatin hydrogels. Since the crystals grow against the hydrogel structure, the influence of the solid content of the gel becomes obvious from the size and morphology of the obtained crystals. The crystals become progressively smaller and have rougher surfaces as the solid content in the hydrogel increases. Moreover, higher solid contents in the hydrogel correlate with increasing imperfection in crystalline constitution on (at least) three length scales: (i) a mesocrystal architecture with increasing imperfection in the precise coalignment of the constituting nanoscale units, (ii) a microscale intracrystalline mosaic structure with subgrains separated by small-angle boundaries or, occasionally, smooth continuous orientation gradients, and (iii) the formation of radial aggregates of crystals on the scale of tens to hundreds of micrometers. For the gel with $2.5 \mathrm{wt} \%$ gelatin, the $\sim 100 \mu \mathrm{m}$ sized crystals constituting the aggregate still show some degree of mutual coalignment, while for the gel with $10 \mathrm{wt} \%$ gelatin, a coorientation can no longer be observed. In order to understand these findings, it is necessary to investigate how an increase in the solid content of the hydrogel affects the physicochemical conditions of the fluid where crystallization occurs.

Physicochemical Conditions at Early Stages of Crystallization. In hydrogels, nucleation and early stages of crystallization occur in confined solutions, within the volume of a pore. In this type of system, the concentration of the reagents, the $\mathrm{pH}$, and the supersaturation continuously evolve in time and space. It has been demonstrated that this evolution depends on the boundary conditions ${ }^{61,62}$ (i.e., the initial concentration of the reagents in the deposits and the length of the diffusion column). Furthermore, it has been stated that the characteristics of this evolution control the supersaturation at which nucleation (of whichever phase) occurs. ${ }^{54,55}$ This supersaturation is referred to as the threshold supersaturation. While in the case of crystallization from free aqueous solution, the critical supersaturation (i.e., the supersaturation at which nucleation rate reaches $1 \mathrm{~cm}^{-3} \mathrm{~s}^{-1}$ ) has a single value for a given system, ${ }^{63}$ the threshold supersaturation, which applies to systems where supersaturation is a function dependent on both time and space, has different values depending on the supersaturation rate
$\left(R_{\beta}\right)$ (i.e., the rate at which the system moves from equilibrium). ${ }^{64}$ It has been demonstrated that higher concentrations of the counter-diffusing reagents and shorter lengths of the diffusion column result in nucleation occurring under higher supersaturation levels (i.e., lead to higher values of the threshold supersaturation). ${ }^{65,66}$ The reason underlying this behavior is a faster change in the concentration profiles along the gel column and, consequently, a higher supersaturation rate.

In the crystal growth experiments conducted in this study, both the concentration of the reagents and the length of the gel column were identical. The optically first visible crystals corresponded to calcite, and the time needed to form them increased significantly with increasing concentration of gelatin in the gel.

A possible explanation for the increase of waiting time by a factor of 5 between the $2.5 \%$ and the $10 \%$ gelatin gel may be a reduced diffusion rate in the denser gels. The diffusion coefficient in the gel $\left(D_{\mathrm{g}}\right)$ relates to the diffusion coefficient in water $\left(D_{\mathrm{w}}\right)$ by the expression:

$$
D_{\mathrm{g}}=D_{\mathrm{w}}\left(\varphi / \tau^{2}\right)
$$

where $\varphi$ and $\tau$ are the porosity and the effective tortuosity of the hydrogel, respectively. ${ }^{67}$ An increase in the solid content of a hydrogel correlates with a decrease of its total porosity and necessarily induces a decrease of the diffusion coefficients compared to those in water. This effect can be enhanced if higher solid contents also determine more complex porosity structures and, consequently, higher tortuosity, for instance, by generating isolated pores, dead-end pore paths, etc. This is the case in gelatin hydrogels, where an organized porosity of greater complexity appears as the gelatin content in the hydrogel increases. Both, the lower percentage of porosity and the more complex structure of porosity will lead to lower diffusion coefficients. Consequently, the counter-diffusion of the reagents must occur at lower rates in hydrogels with higher gelatin contents and, as a result, longer diffusion times should be required to reach identical supersaturation (of whichever phase) in the different gels. To test this hypothesis, we conducted the single-component diffusion experiments (with either $\mathrm{Ca}^{2+}$ or $\mathrm{HCO}_{3}{ }^{-}$, respectively) in the different gels. The result, however, clearly contradicts the hypothesis of a diffusion-related delay. Given the measured waiting times, the diffusion through the gel column clearly delivered much larger amounts of $\mathrm{Ca}^{2+}$ and $\mathrm{HCO}_{3}{ }^{-}$to the location of crystallization at early stages of calcite formation, than in the gel with less gelatin. For the denser gels, the calculated calcite nominal supersaturation from the ionic concentrations at the early stage of calcite crystallization is even more than ten times higher than for the gel with $2.5 \%$ gelatin (Table 2 ). None of the gels showed any sign of turbidity. Turbidity would have been a clear sign for the formation of a finely dispersed precursor, ${ }^{48-50}$ which, if present, would provide a sink for the calcium carbonate in solution. The absence of turbidity, however, does also not rule out the presence of dilute nanoscale precursor particles.

The experiments with higher gelatin content lead to much more disordered structures of the calcite aggregates (with respect to a perfect single crystal). In accordance with the nominal supersaturation data, the early stages of calcite crystallization take place significantly farther away from equilibrium conditions as the gelatin content in the hydrogel increases. Therefore, an important influence of the gel structure on the nucleation and growth of the calcite must be concluded. Either, the denser gel structure leads to a dramatic increase of the critical super- 
saturation for calcite formation, and/or it induces a dramatic increase in the formation of a nanometric solid precursor. For the latter, however, we see no evidence so far.

In order to explain the potential effect of the gel solid content on supersaturation at early crystallization stages, other characteristics of the porosity than its complexity and influence in diffusivity have to be considered. A most relevant feature of porosity is the size of the pores. In gelatin hydrogels, this size clearly decreases as the solid content increases, mainly due to the increase of the pore wall thickness and the formation of subpores within bigger pores. Nucleation and early crystallization stages in gels occur in the bulk of aqueous solutions confined in the volume of pores. It has been demonstrated that diminishing the pore size in silica gel and gelatin lowers the nucleation probability. ${ }^{65,68,69}$ This can be easily explained, considering that nucleation occurs as a result of fluctuations that bring together a sufficiently large number of ions to exceed the critical size. $^{70}$ In the case of sparingly soluble phases, such as calcium carbonate polymorphs, the number of ions in a given volume is small, even if supersaturation is high. Diminishing the volume of solution cells makes this number smaller and, consequently, nucleation less probable. This consideration is further supported by the fact that the number of visible crystals at the waiting times specified in Table 1 decreases significantly with increasing gelatin content of the hydrogels. We thus conclude that higher supersaturation levels are required for crystallization from solutions confined to smaller pores. An increase of the supersaturation increases the number of ions available in the volume of a small pore, facilitating the nucleation process.

Crystallization Process. As explained above, higher gelatin content in the hydrogels correlate with a lower number of calcite crystals per volume of hydrogel, smaller crystal sizes, and a predominance of spherulitic aggregates with respect to single crystals in the precipitates. Crystallization occurring at significantly higher supersaturation can explain the variation in the crystal sizes and the predominance of aggregates as the hydrogel gets heavier.

It is notorious that crystallization in hydrogels always occurs under high (at least nominal) supersaturations. ${ }^{40,42,65,66,71}$ Under these conditions, neither nucleation nor growth follows classical paths. Nonclassical nucleation can lead to the formation of amorphous or metastable phases. ${ }^{72}$ Although the formation of a nanometric phase prior to calcite in these experiments cannot be confirmed nor discarded by our experiment, calcite, the stable $\mathrm{CaCO}_{3}$ polymorph at $15^{\circ} \mathrm{C}$, was always the main component of the precipitate and the first phase that was optically detected. Moreover, the amount of vaterite found was higher in the precipitate formed in the hydrogel with the lower gelatin content (i.e., where nominal supersaturation at early crystallization stages should be lower). The reason for the formation of this higher amount of vaterite in the later stages of crystallization in lighter gelatin hydrogels and its inhibition in heavier hydrogels are unclear and cannot be connected to an effect of the supersaturation parameter.

The classical layer-by-layer growth mechanism, which involves the integration of atoms or molecules onto energetically favorable sites on crystal surfaces, ${ }^{73,74}$ is not likely to be efficient when mass transfer is limited, the aqueous solution is confined in the small volume of pores, and supersaturation is very high. Moreover, the peculiar characteristics of the calcite crystals grown in hydrogels with high gelatin content cannot really be explained by a layer-by-layer growth mechanism. In this case an alternative scenario can be contemplated. It is generally accepted $^{1,16,25,27}$ that under these conditions (solution confined in small pores and high supersaturation), crystal growth occurs through a specific mechanism involving the oriented attachment of short-range ordered (subcritical) clusters due to dipolar forces. ${ }^{75-78}$ The presence of large amounts of these clusters would also reduce the activity of the calcium and carbonate ions and, hence, the supersaturation compared to the nominal supersaturation, which we calculated from the results of the single-component diffusion experiments. The driving force for particle attachment would be the reduction of the surface free energy that results from the crystallographic fusion of aggregated nanoparticles. ${ }^{1,16-19,23,37}$ This aggregation mechanism leads to the formation of an assembly of nanometric, basically equally oriented sub-blocks. This mechanism can therefore explain the characteristics of the calcite crystals obtained in this work in hydrogels with gelatin content $\geq 5 \mathrm{wt} \%$. During the aggregation process, a small amount of hydrogel is incorporated in between the assembled nanoparticles. This amount directly relates to the concentration of gelatin solid in the hydrogel and the increased thickness of the hydrogel pore walls. Larger incorporations may also be favored by both higher viscosities and supersaturations in the growth medium. The characteristics of calcite crystals formed in hydrogels with $2.5 \mathrm{wt} \%$ gelatin content does not support the idea that aggregation played a dominant role in their growth. These crystals also grew under high supersaturation, although it is not as high as that for the crystals formed in hydrogels with gelatin content $\geq 5$ wt $\%$, and incorporated some gelatin. Our results point to the switch from classical to nonclassical growth mechanisms, depending on subtle relationships between supersaturation, growth medium viscosity, and hydrogel incorporation during growth.

It is important to know that the dipolar forces involved in the oriented attachment of crystal sub-blocks strongly vary with distance. Consequently, mesocrystals formed in more viscous, thicker wall-pored hydrogels should show a less perfect coorientation of their nanometric building blocks. This is consistent with our EBSD observations, which show that the mosaic spread within calcite crystal individuals grown in hydrogels with $10 \mathrm{wt} \%$ gelatin content is almost twice as high as that measured in calcite crystals formed in hydrogels with 2.5 wt $\%$ gelatin.

It is also interesting that the mosaic spread measured within crystal individuals grown in $10 \mathrm{wt} \%$ gelatin hydrogels is not homogeneous but increases from core to rim. To attain a perfect coalignment of the nanoparticles, the hydrogel has to be expelled from in between the particles. This process will necessarily involve the deformation of the hydrogel pore walls as they are squeezed by the attaching particles, and it will give an increase of the viscosity of the growth medium at the front of the growing mesocrystal aggregate, as the hydrogel is expelled from inner to outer regions. The direct consequence is a higher hydrogel incorporation in the rim region of the crystal aggregates, which in turn leads to the higher particle misorientation in the rim in comparison to that in the core of the mesocrystals, as evidenced by the EBSD measurements.

\section{CONCLUSIONS}

$\mathrm{CaCO}_{3}$ was crystallized in gelatin hydrogels with different solid contents. Our results show that this parameter strongly influences the crystallization of carbonate crystals. Regarding early stages of crystallization, the nominal supersaturation markedly increased with the gelatin content of the hydrogel. However, this parameter had little effect on the $\mathrm{CaCO}_{3}$ 
polymorphic selection, with the precipitate always consisting of mainly calcite. With regard to the characteristics of calcite crystals, we observed that with increasing gelatin concentrations in the hydrogel, the calcite (1) showed smaller crystals sizes, rougher surfaces, more complex growth morphologies, and a tendency to develop as crystal aggregates rather than as single crystals, (2) incorporated increasing amounts of gelatin during their growth, and (3) showed an increasing degree of internal structuring with an orientational mosaic spread that increases rapidly with increasing gelatin concentration of the hydrogel. For low gelatin concentrations, we find a certain degree of mutual coalignment of the $\sim 100 \mu \mathrm{m}$ sized crystals forming the macroscopic (submillimeter) aggregates, while for $10 \mathrm{wt} \%$ gelatin concentration in the gel a spherulite-like radial configuration of the macroscopic aggregates prevails. The internal mosaic structure within the individual crystals of the aggregates comprises both microscale mosaic blocks separated by small-angle boundaries as well as an underlying mesocrystalline structure: the microcrystals are built of imperfectly coaligned nanoscale units.

The influence of the hydrogel solid content on the crystallization process and the characteristics of calcite crystals arises from changes in hydrogel porosity characteristics. The changes in porosity (1) induce changes in the diffusivity and, in turn, (2) in the evolution of the physicochemical conditions of the gel during counterdiffusion, they affect the early stages of crystallization by varying the volume of aqueous solution available within the hydrogel pores, and (3) induce the transition from a classical layer-by-layer crystal growth mechanism to an aggregation mechanism as the hydrogel becomes more viscous, thereby promoting the formation of mesocrystals.

\section{ASSOCIATED CONTENT}

\section{S Supporting Information}

SEM images of crushed calcite crystals, Raman spectrum of powdered skin pork gelatin, TGA curves of calcite aggregates, and a table of crystallite size comparison. This material is available free of charge via the Internet at http://pubs.acs.org.

\section{AUTHOR INFORMATION}

\section{Corresponding Authors}

*E-mail: f.nindiyasari@lmu.de. Tel: +49-890-2180-4354.

*E-mail: lfdiaz@geo.ucm.es.

\section{Notes}

The authors declare no competing financial interest.

\section{ACKNOWLEDGMENTS}

This research is part of the German-Spanish joint Acciones Integradas program (AIB2010 DE-0008), DAAD-50749739. F.N. is grateful for a stipendium by KAAD. E.G. is supported by Deutsche Forschungsgemeinschaft, DFG Grant GR-1235/9-1. This research was partially funded by project CGL2010-20134C02-01 (MECC-Spain). We are indebted to Sabino Veintemillas-Verdaguer (ICMM, CSIC) for his advice and help in the characterization of the hydrogel porosity. We sincerely thank Eugenio Baldonedo from the National Microscopy Centre (ICTS), Emilio Matesanz from the X-ray Diffraction Central Service, and the staff from the Laboratory of Geochemistry and Environmental Analysis of the Complutense University (UCM) for technical support and assistance. We are also grateful to Laura Tormo and Cristina Paradela for enabling Raman measurements at the MNCN, CSIC (Madrid, Spain).

\section{REFERENCES}

(1) Meldrum, F.; Cölfen, H. Chem. Rev. 2008, 108, 4332-4432.

(2) Asenath-Smith, E.; Li, H.; Keene, E. C.; Seh, Z. W.; Estroff, L. A. Adv. Funct. Mater. 2012, 22, 2891-2914.

(3) Kobayashi, I.; Samata, T. Mater. Sci. Eng. 2006, 26, 692-698.

(4) Falini, G.; Albeck, S.; Weiner, S.; Addadi, L. Science 1996, 271, 6769.

(5) Cusack, M.; Freer, A. Chem. Rev. 2008, 108, 4433-4454.

(6) Griesshaber, E.; Schmahl, W. W.; Neuser, R.; Pettke, T.; Blüm, M.; Mutterlose, J.; Brand, U. Am. Mineral. 2007, 92, 722-734.

(7) Levi-Kalisman, Y.; Fallini, G.; Addadi, L.; Weiner, S. J. Struct. Biol 2001, 135, 8-17.

(8) Zhou, J.; Zhou, M.; Caruso, R. A. Langmuir 2006, 22, 3332-3336.

(9) Kim, U.; Park, J.; Li, C.; Jin, H.; Valluzzi, R.; Kaplan, D. L. Biomacromolecules 2004, 5, 786-792.

(10) Xie, M.; Olderøy, M. Ø.; Andreassen, J. P.; Selbach, S. M.; Strand, B. L.; Sikorski, P. Acta Biomaterialia 2010, 6, 3665-3675.

(11) Annabi, N.; Nichol, J. W.; Zhong, X.; Ji, C.; Koshy, S.; Khademhosseini, A.; Dehghani, F. Tissue Eng. 2010, 16, 371-383.

(12) McCauley, J. W.; Roy, R. Am. Mineral. 1974, 59, 947-963.

(13) García-Ruíz, J. M.; Amorós, J. L. J. Cryst. Growth 1981, 55, 379383.

(14) Fernández-Diaz, L.; Putnis, A.; Prieto, M.; Putnis, C. V. J. Sediment. Res. 1996, 66, 482-491.

(15) Grassmann, O.; Muller, G.; Löbmann, P. Chem. Mater. 2002, 14, $4530-4535$

(16) Grassmann, O.; Neder, R. B.; Putnis, A.; Löbmann, P. Am. Mineral. 2003, 88, 647-652.

(17) Grassmann, O.; Löbmann, P. Chem.-Eur. J. 2003, 9, 1310-1316.

(18) Sethmann, I.; Putnis, A.; Grassmann, O.; Löbmann, P. Am. Mineral. 2005, 90, 1213-1217.

(19) Huang, Y.; Buder, J.; Cardoso-Gil, R.; Prots, Y.; Carrillo-Cabrera, W.; Simon, P.; Kniep, R. Angew. Chem., Int. Ed. 2008, 47, 8280-8284.

(20) Yuwono, V. M.; Burrows, N. D.; Soltis, J. A.; Penn, R. L. J. Am. Chem. Soc. 2010, 132, 2163-2165.

(21) Song, R.-Q.; Cölfen, H. Adv. Mater. 2010, 22, 1301-1330.

(22) Seto, J.; Ma, Y.; Davis, S. A.; Meldrum, F.; Gourrier, A.; Kim, Y.-Y.; Schilde, U.; Sztucki, M.; Brughammer, M.; Maltsev, S.; Jäger, C.; Cölfen, H. Proc. Natl. Acad. Sci. U.S.A. 2012, 109, 3699-3704.

(23) Cölfen, H.; Antonietti, M. Angew. Chem., Int. Ed. 2008, 44, 55765591.

(24) Song, R.-Q.; Cölfen, H. Cryst. Eng. Comm. 2011, 13, 1249-1276.

(25) Gebauer, D.; Coelfen, H. Nano Today 2011, 6, 564-584.

(26) Gebauer, D.; Völkel, A.; Cölfen, H. Science 2008, 322, 18191822.

(27) Cölfen, H. Nat. Mater. 2010, 9, 960-961.

(28) Dey, A.; Bomans, P. H. H.; Müller, F. A.; Will, J.; Frederik, P. M.; de With, G.; Sommerdijk, N. A. J. M. Nat. Mater. 2010, 9, 1010-1014.

(29) Demichelis, F.; Raiteri, P.; Gale, J. D.; Quigley, D.; Gebauer, D. Nat. Commun. 2011, 2, 1-8.

(30) Picker, A.; Matthias, K.; Seto, J.; Gebauer, D.; Cölfen, H. Z. Kristallogr. 2012, 227, 744-757.

(31) Vielzeuf, D.; Floquet, N.; Chatain, D.; Bonneté, F.; Ferry, D.; Garrabou, J.; Stolper, E. M. Am. Mineral. 2010, 95, 242-248.

(32) Floquet, N.; Vielzeuf, D. Cryst. Growth Des. 2012, 12, 4805-4820.

(33) Seidl, B. H.; Reisecker, C.; Hild, S.; Griesshaber, E.; Ziegler, A. Z. Kristallogr. 2012, 227, 777-792.

(34) Kelm, K.; Goetz, A.; Sehrbrock, A.; Irsen, S.; Hoffmann, R.; Schmahl, W. W. Z. Kristallogr. 2012, 227, 758-765.

(35) Schmahl, W. W.; Griesshaber, E.; Kelm, K.; Ball, A.; Goetz, A.; Xu, D.-Y. Z. Kristallogr. 2012, 227, 604-611.

(36) Schmahl, W. W.; Griesshaber, E.; Kelm, K.; Goetz, A.; Guntram, J.; Ball, A. Z. Kristallogr. 2012, 227, 793-804.

(37) Grassmann, O.; Löbmann, P. Biomaterials 2004, 25, 277-282.

(38) Fernández-Díaz, L.; Astilleros, J. M.; Pina, C. M. Chem. Geol. 2006, 225, 314-321.

(39) Hanying, L.; Estroff, L. A. J. Am. Chem. Soc. 2007, 129, 54805483. 
(40) Sánchez-Pastor, N.; Gigler, A. M.; Cruz, J. A.; Park, S.-H.; Jordan, G.; Fernández-Díaz, L. Cryst. Growth Des. 2011, 11, 3081-3089.

(41) Dorwee, J. R.; Boskey, A. L.; Estroff, L. A. Cryst. Eng. Comm. 2012, $14,5681-5700$.

(42) Helbig, U. J. Cryst. Growth 2008, 310, 2863-2870.

(43) Minchin, D. Mar. Biol. 1987, 95, 139-145.

(44) Hahn, S.; Rodolfo-Metalpa, R.; Griesshaber, E.; Schmahl, W. W.; Buhl, D.; Hall-Spencer, J. M.; Baggini, C.; Fehr, K. T.; Immenhauser, A. Biogeosciences 2012, 9, 1897-1914.

(45) Rijssel, M.; Giesken, W. W. C. J. Sea Res. 2002, 48, 17-27.

(46) Schouten, S.; Ossebaar, J.; Schreiber, K.; Kienhuis, M. V. M; Langer, G.; Benthien, A.; Bijma. J. Biogeosciences 2006, 3, 113-119.

(47) Parkhurst, D. L.; Appelo, C. A. J. In User's Guide to PHREEQC, U.S. Geological Survey Water Resources Investigations Report 99-4259; U.S. Geological Survey: Washington, DC. 1999.

(48) Wang, Y.-W.; Kim, Y.-Y.; Stephens, C. J.; Meldrum, F. C.; Christenson, H. K. Cryst. Growth Des. 2012, 12, 1212-1217.

(49) Huang, S.-C.; Naka, K.; Chujo, Y. Langmuir 2007, 23, 1208612095.

(50) Loste, E.; Wilson, R. M; Seshadri, R.; Meldrum, F. C. J. Cryst. Growth 2003, 254, 206-218.

(51) Rutt, H. N.; Nicola, J. H. J. Phys. C: Solid State Phys. 1974, 7, $4522-4528$.

(52) Gunasekaran, S.; Anbalagan, G.; Pandi, S. J. Raman Spectrosc. 2006, 37, 892-899.

(53) Soldati, A. L.; Jacob, D. E.; Wehrmeister, U.; Häger, T.; Hofmeister, W. J. Raman Spectrosc. 2006, 39, 525-536.

(54) Vandenabeele, P.; Moens, L.; Edwards, H. G. M.; Dams, R. J. Raman Spectrosc. 2000, 31, 509-517.

(55) Barnard, W.; Waal, D. J. Raman Spectrosc. 2006, 37, 342-352.

(56) Nelson, W. H.; Carey, P. R. J. Raman Spectrosc. 1981, 31, 326328.

(57) Veronelli, M.; Zerbi, G.; Stradi, R. J. Raman Spectrosc. 1995, 26, 683.

(58) Fujimori, K.; Sakamoto, A.; Tasumi, M. Macromol. Symp. 2004, 205, 33-46.

(59) Goetz, A. J.; Steinmetz, D. R.; Griesshaber, E.; Zaefferer, S.; Raabe, D.; Kelm, K.; Irsen, S.; Sehrbrock, A.; Schmahl, W. W. Acta Biomater. 2011, 7, 2237-2243.

(60) Griesshaber, E.; Schmahl, W. W.; Ubhi, H. S.; Huber, J.; Nindiyasari, F.; Maier, B.; Ziegler, A. Acta Biomater. 2013, in press.

(61) Henisch, H. K.; Garcia-Ruiz, J. M. J. Cryst. Growth 1986, 75, 195202.

(62) Henisch, H. K.; Garcia-Ruiz, J. M. J. Cryst. Growth 1986, 75, 203211.

(63) Walton, A. G. Nucleation in Liquids and Solutions. In Nucleation; Zottlemoyer, A. C., Ed.; Marcel Dekker: New York, 1969, pp 225-307.

(64) Prieto, M.; Fernández-Díaz, L.; López-Andrés, S. J. Cryst. Growth

1991, 108, 770-778.

(65) Putnis, A.; Prieto, M.; Fernández-Díaz, L. Geol. Mag. 1995, 132, $1-13$.

(66) Prieto, M.; Putnis, A.; Fernández-Díaz, L.; López-Andrés, S. J. Cryst. Growth 1994, 142, 225-235.

(67) Oelkers, E. H. Rev. Mineral. 1996, 34, 131-191.

(68) Halberstadt, E. S.; Henisch, H. K.; Nickl, J.; White, E. W. J. Colloid Interface Sci. 1969, 29, 469-471.

(69) Henisch, H. K. Crystals in Gels and Liesegang Rings; Cambridge University Press: Cambridge, 1988.

(70) De Yoreo, J. J.; Vekilov, P. G. Rev. Mineral. Geochem. 2003, 54, 57-93.

(71) Prieto, M.; Fernández-Díaz, L.; López-Andrés, S. J. Cryst. Growth 1989, 98, 447-460.

(72) Chernov, A. A.Givargizov, E. J.Bagdasarov, K. S.Kuznetsov, V. A. Modern Crystallography III: Crystal Growth; Springer: Berlin, 1984.

(73) Kossel, W. Nachr. Akad. Wiss. Göttingen, Math.-Phys. Kl., 2 1927, 135-143.

(74) Burton, W. K.; Cabrera, N.; Frank, F. C. Phil. Trans. R. Soc., A 1951, 243, 299-358.
(75) Niederberger, M.; Cölfen, H. Phys. Chem. Chem. Phys 2006, 8, 3271-3287.

(76) Lee, E. J. H.; Ribeiro, C.; Longo, E.; Leite, E. R. J. Phys. Chem. B 2005, 109, 20842-20846.

(77) Zhang, Q.; Liu, S. H.; Yu, S. H. J. Mat. Chem. 2009, 19, 191-207. (78) Yao, Q. Z.; Guan, Y. B.; Fu, S. Q. Eur. J. Mineral. 2012, 24, 519526. 